Please cite this article as:

P. López-Varo, J. A. Jiménez-Tejada, O. Marinov, J.E. Carceller, C.H. Chen, M. J. Deen, Boundary condition model for the simulation of organic solar cells, Organic Electronics, (2017), 48, 85-95.

(C)2017. This manuscript version is made available under the CC-BYNC-ND 4.0 license http://creativecommons.org/licenses/by-nc-nd/4.0/

Digital Object Identifier: 10.1016/j.orgel.2017.05.046

Source: http://www.sciencedirect.com/science/article/pii/S1566119917302434 


\title{
Boundary condition model for the simulation of organic solar cells
}

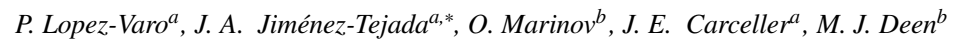

${ }^{a}$ Departamento de Electrónica y Tecnología de los Computadores, CITIC-UGR, Facultad de Ciencias, Universidad de Granada, Granada 18071, Spain
${ }^{b}$ Department of Electrical and Computer Engineering, McMaster University, Hamilton, ON L8S 4K1, Canada

\begin{abstract}
Organic solar cells (OSCs) are promising photovoltaic devices to convert solar energy into electrical energy. Their many advantages such as lightweight, flexibility and low manufacturing costs are intrinsic to the organic/polymeric technology. However, because the performance of OSCs is still not competitive with inorganic solar cells, there is urgent need to improve the device performance using better designs, technologies and models. In this work, we focus on the developing an accurate physics-based model that relates the charge carrier density at the metal-organic boundaries with the current density in OSCs using our previous studies on singlecarrier and bipolar diodes. The model for the boundary condition of the charge carrier density at the interfaces of OSCs follows a power-law function with the current density, both in dark and under illumination, and simulated current-voltage characteristics are verified with experimental results. The numerical simulations of the current-voltage characteristics of OSCs consider wellestablished models for the main physical and optical processes that take place in the device: light absorption and generation of excitons, dissociation of excitons into free charge carriers, charge transport, recombination and injection-extraction of free carriers. Our analysis provides important insights on the influence of the metal-organic interfaces on the overall performance of OSCs. The model is also used to explain the anomalous S-shape current-voltage curves found in some experimental data.
\end{abstract}

Keywords: Organic solar cells, Modeling, Boundary conditions for simulation, Charge carrier density at interfaces

\section{Introduction}

Organic-polymeric (hereafter, we use organic to mean both organic and polymeric) solar cells (OSCs) can play an important role in the future photovoltaics market. OSCs have important advantages such as simplicity of the production processes, the low processing temperatures, printing over large area and flexible substrates, low weight, and low environmental impact that are intrinsic to organic materials. Despite these advantages, the economic potential of OSCs is not realized because presentday conversion efficiencies are not high enough for their largescale use in the energy industry. Therefore, there are much research and technology development efforts to bring this technology up to the required level to be economically viable for widespread use.

Currently, much research and technology development of OSCs is focused on improving efficiency of stable, longlifetime devices [1-4]. An attractive way to improve the performance of OSCs is through simulation and modeling which allow for both reduction in both costs and development time of the technology. For accurate prediction of OSCs performance, the main physical-chemical mechanisms: light propagation in the materials, creation and dissociation of excitons into electrical charges, and drift-diffusion transport of the charge carriers,

\footnotetext{
${ }^{*}$ Corresponding author

Email addresses: pilarlopez@ugr.es (P. Lopez-Varo), tejada@ugr.es (J. A. Jiménez-Tejada), omarinov@yahoo.com (O. Marinov), jcarcell@ugr.es (J. E. Carceller), jamal@mcmaster.ca (M. J. Deen)
}

must be incorporated into the numerical simulator. Previously, in several excellent works, the understanding of each mechanism and to interactions between different mechanisms was considered [5-13]. The backbone of these models is the set of transport equations: the continuity, Poisson and drift-diffusion current equations (see equations (4)-(12) later). It must be noted that the role that metal-organic (MO) interfaces play in OSCs performance is embedded as boundary conditions for the solution of this set of differential equations (DEs). The solution of DEs depends on boundary conditions, although DEs themselves do not provide for the boundary conditions. Therefore, boundary conditions for simulation of OSCs require special attention. Actually, the aforementioned simulation studies differ mainly in the choice of the boundary conditions for the electron and hole concentrations at the interfaces.

The charge carrier density at the MO interface can be governed by several physical-chemical mechanisms such as thermionic emission, tunneling, reduction-oxidation (redox) reactions, trap-assisted recombination, and band bending due to effects such as Fermi-level pinning or dipoles at the interface that control the injection and extraction of charge [14, 15]. The combinations and proportions of these different effects and mechanisms complicate the task of modeling MO interfaces. Different models for the extraction and injection of charge have been developed in order to find proper boundary conditions for the free charge density or the electric field at the MO interface [16-21]. In practice, approximations of these models are commonly used in order to reduce the high computational volumes of numerical DE solvers. 
In OSC modeling and simulation, typical boundary conditions at the material interfaces consider either constant charge density, determined by Boltzmann statistics in thermionic emission over energy barriers [22, 23], or constant surface recombination velocity at metal-organic interfaces, which inherently implies a linear relation between the interface charge and the current density. In particular, the linear relation of the electron, $n$ (or hole, $p$ ) density with the current density $J$ at constant surface recombination velocity $S$ is usually written as $n=-J / S+n_{0}$ (or $\left.p=-J / S+p_{0}\right)[5,24]$, where $n, p$ are the electron and hole densities at the interfaces and $n_{0}, p_{0}$ are their respective values at equilibrium. These simple approximations (constant interface charge and constant surface recombination velocity) provide easy means in the modeling of OSCs, but the approximations are rarely used for consistency and almost never for accuracy.

Recently, we have proposed a more accurate relation between the free charge density at the MO interfaces and the current density flowing through these interfaces. In general, the relation is based on a power-law dependence between the charge and the current. This model was initially proposed for single-carrier, and then, for bipolar organic diodes in order to interpret their current-voltage characteristics in dark $[25,26]$. We have observed that the free charge density at the injecting contact (for example at $x=0)$ of a single-carrier diode $(n(0)=n(x=0)$ or $p(0)=p(x=0))$ is related to the density of the following current by a power-law function,

$$
n(0)[, p(0)]=K_{1} J^{m}+K_{2}=\left[\left(J / J_{D}\right)^{m}+1\right] K_{2},
$$

where $m$ is a parameter that depends on the organic material, $K_{1}$ is a parameter related to the energy barrier at the injecting contact and $K_{2}$ is a parameter that models a flat region for the charge density at the interface at low values of the current density. $J_{D} \equiv\left(K_{2} / K_{1}\right)^{1 / m}$ is the corner current density at which (1) transits from a constant $K_{2}$ to a power law function $K_{1} J^{m}$. In diffusion-dominated transport, at bias close to the diode's builtin voltage, the charge density at the contact is almost a constant independent of the current. The value of this charge is given by the parameter $K_{2} . K_{2}$ also provides information about the existence of thermal carriers, the doping of the semiconductor or traps close to the interface [27, 28]. Later, we will show that $K_{2}$ elevates under illumination.

The power-law expression (1) incorporates the above mentioned Boltzmann and linear $J-n$ (or $J-p$ ) approximations, with $m=0$ and $m=1$, respectively. It also takes into account possible recombination mechanisms at the interfaces through $m \neq 0$ [26]. After experimental observations in OSCs, powerlaw relations of the type $J \propto n^{2.6}$ were found between the dark current density with the charge stored in the active layer of an OSC, and between the total photogenerated current with the charge stored at open circuit $[29,30]$. This would agree with our power-law expression (1). Overall, the power-law model for the interface charge as a function of the current density is very consistent with experimental data for $J-V_{A C}$ curves of organic diodes, including OSCs in dark [26] $\left(V_{A C}\right.$ is the external applied voltage). Using the extraction procedures developed in these analyses, we will show later that the power-law model remains valid when OSCs are under illumination, just $K_{2}$ is different and higher under illumination.

In this work, we study the effect of the illumination on the power-law model (1) for the charge-current dependence at metal-organic interfaces of OSCs. We also assess the impact of this interface effects on the $J-V_{A C}$ curves of the OSCs. The objective is to find a proper model for the values of the free charge carrier densities at the anode and cathode of OSCs that can be used in simulators of these devices. Bearing this in mind, Section 2 summarizes the optical and electrical models used for the simulation of the active layer of OSCs [7, 31]. In Section 3 , we adapt the boundary conditions at the metal-organic interfaces to OSCs. In Section 4 , numerical $J-V_{A C}$ curves are simulated and comparisons with experimental data are given. A procedure to extract the values of our model parameters is also proposed along with these comparisons. In darkness, $J-V_{A C}$ curves are analyzed both in forward and reverse regimes, corresponding to the injection from both contacts. In these situations, our previously developed model for single carrier diodes is completely valid. Under illumination, the contact model for OSCs is finally arranged considering previous experimental observations [29, 30] and suggestions for boundary conditions in simulation [32]. In this definitive arrangement, the charge density at the interface increases when the intensity of the incident light increases, following again the power-law function. The main conclusions are provided in Section 5.

\section{Electrical and optical models for the active layer of OSCs}

A typical organic solar cell is a multilayer structure with a metallic cathode of a low work function, an anode of a high work function, an organic active layer where the absorbed light generates photo-charge. OSC also has a substrate that supports the entire structure. The OSC active layer is composed of two semiconductor compounds that allow for the separation of photogenerated electron-hole pairs. An acceptor compound (usually fullerene) collects and transports photogenerated electrons. A donor compound (usually a polymer) collects and transports photogenerated holes. These two compounds can be separated by a well-defined interface, giving rise to the socalled bilayer OSC, or can be blended together in the case of the bulk-heterojunction (BHJ) solar cell. The latter BHJ OSC achieves greater efficiencies than the former bi-layer OSC. For this reason, the BHJ structure is analyzed in this work.

We use the effective medium approach in the simulation of the active layer of the BHJ OSC, in which the active layer is considered as a uniform material with an effective band gap $E_{G}$ defined as the energy difference between the lowest unoccupied molecular orbital (LUMO) of the acceptor material ( $\left.L U M O_{a c c}\right)$ and the highest occupied molecular orbital (HOMO) of the donor layer $\left(\mathrm{HOMO}_{\text {donor }}\right)$. A simplified energy-level diagram of a BHJ solar cell is shown in Fig. 1. The difference in the work functions of the contacts creates an internal built-in voltage $V_{b i}$ that facilitates the transport of the photogenerated charge carriers. The energy barriers that electrons and holes see from the contacts towards the organic semiconductor are higher in reverse operation of OSCs (when the applied voltage $V_{A C}$ is 
$\left.V_{A C}<0\right)$ than in forward operation $\left(V_{A C}>0\right)$. This asymmetry of contact work functions and barriers creates an asymmetry in the current-voltage curves of OSCs between forward and reverse operations under dark condition.
(1) Light

(2) Exciton generation

(3) Exciton diffusion
(4) Charge separation

(5) Charge transport

(6) Charge collection

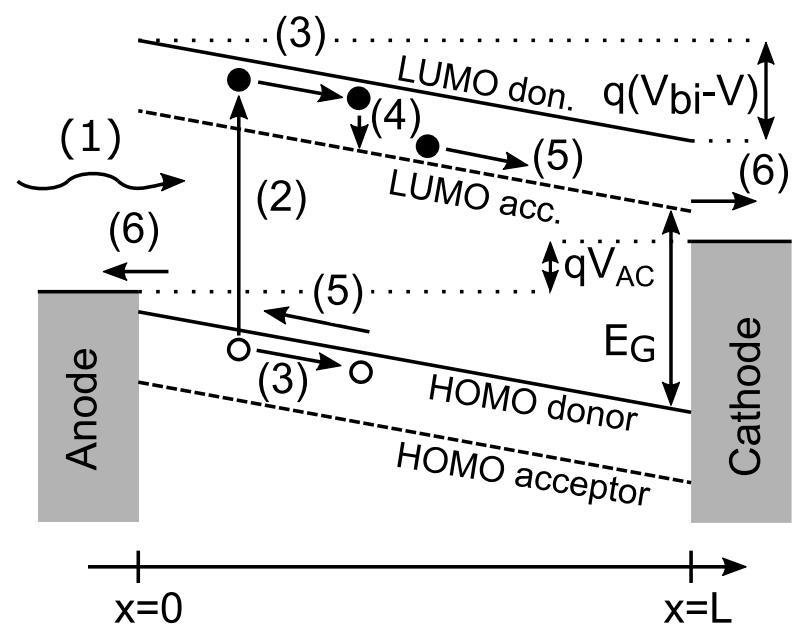

Figure 1: Schematic energy diagram of a BHJ solar cell indicating the main mechanisms that take place for the transformation of the sunlight in electrical current (recombination processes are not shown).

Under illumination, the current-voltage characteristic of an OSC is the result of simultaneously occurring optical and electrical mechanisms (Fig. 1) of: (1) optical propagation and photon absorption, (2) exciton (or bound electron-hole pair) formation, (3) exciton migration, (4) exciton dissociation at a donor/acceptor interface, (5) charge transport and bimolecular recombination in the semiconductor and (6) charge extraction at the metal-organic extracting contacts.

\subsection{Optical models for the active layer of OSCs}

The photocurrent density in an OSC is the flow of charge carriers generated in the active layer of the device, which depends on the number of generated excitons. The number of excitons varies with the optical power and the photon energy. The energy of the exciton is equal to the energy of the absorbed photon $h c / \lambda$, where $\lambda$ is the wavelength of the incident light. Thus, the exciton can be seen as a non-electromagnetic carrier with energy $h c / \lambda$ in the organic material. The propagation and absorption of photons in the layers of OSC is usually calculated by the so-called Transfer Matrix Method (TMM) [8, 13, 33, 34]. In TMM, the photon energy and propagation are represented by the electric field wave of the photon electromagnetic wave. By considering absorption parameters and refractive indices of materials, as well as the intensity spectrum of the incident light, TMM determines the distribution $Q(x, \lambda)$ of the absorbed light.

To dissociate an exciton into electron-hole pair, the exciton energy must be large enough to bring a localized electron from the $H O M O_{\text {donor }}$ to the $L U M O_{a c c}$ of the organic material. Thus, only excitons that absorb photons with wavelength $\lambda<\lambda_{\text {max }} \approx h c /\left|L U M O_{a c c}-H O M O_{\text {donor }}\right|$ contribute to photocharge and photo-current on OSCs. Using $\lambda_{\max }$ as an integration limit of the absorbed light distribution $Q(x, \lambda)$ the spatial distribution of excitons, termed as exciton generation rate, is $G_{\text {exciton }}(x)=\int_{0}^{\lambda_{\max }} Q(x, \lambda) /(h c / \lambda) d \lambda$. TMM is especially useful when the thickness of the OSC layers is below a critical value, as optical interference can appear in the system, and one can eventually maximize the absorbed light (and $G_{\text {exciton }}$, thereof) in the active layer of the OSC.

\subsection{Opto-Electrical Models for the active layer of OSCs}

In a donor-acceptor blend, the photon is absorbed mainly in the donor semiconductor, usually a polymer layer of high efficient light absorption. Excitons diffuse through the donor material until they reach a donor-acceptor interface, where the exciton dissociates in an electron-hole pair (polaron), because the electron is transferred from the exciton to the acceptor material, while the hole remains in the donor material. Since separated in different materials, the electron and hole can approach each other by Brownian motion only up to a distance $d$, which is not zero. They have a chance to recombine by back electron transfer with a probability $\kappa$, or they are definitively dissociated with a dissociation probability $P$. Hilczer and Tachiya extended Onsager model of recombination [35] and calculated the dissociation probability $P=1-\kappa[36]$, where $\kappa$ is given by Eqs. (5)-(7) in [36].

As the time progresses, the electron-hole separation in surviving pairs increases. In this case, recombination between electron and holes coming from different pairs is more probable. The kinetics of this bulk-recombination phase follows a second order kinetics, where the recombination rate $R$ is assumed to be the bimolecular recombination rate [36-38]:

$$
R=\gamma\left(n p-n_{i}^{2}\right)
$$

where $\gamma$ is the bulk rate constant. The bulk rate constant $\gamma$ proposed by Hilczer and Tachiya incorporates the fact that an electron and a hole recombine at a nonzero separation with a finite intrinsic recombination rate and in the presence of an external electric field (see Eqs. (14)-(16) in [36]). In the limit case of zero separation, the expression of $\gamma$ coincides with Langevin's result $\gamma=q\left(\mu_{n}+\mu_{p}\right) /\left(\varepsilon_{0} \varepsilon_{r}\right)$, where $\mu_{n}$ and $\mu_{p}$ are the electron and hole mobilities, respectively, and $\varepsilon_{0}$ and $\varepsilon_{r}$ are the vacuum and relative permittivity of the material, respectively [37].

Then, the net rate of generated free carriers $U$ can be written as

$$
U=G-R=P G_{\text {exciton }}-R
$$

where the charge generation rate is $G=P G_{\text {exciton }}$.

\subsection{Electrical models for the active layer of OSCs}

Once the free electrons and holes are created in the active layer of the OSC they must be transported towards their respective extracting contacts, in order an electrical current to flow. 
The electrical behavior of the OSC active layer is governed by the drift-diffusion model, including also Poisson equation and the continuity equations for electrons and holes. The Poisson equation is

$$
\partial F / \partial x=q\left(p-n+N_{D}-N_{A}\right) /\left(\varepsilon_{0} \varepsilon_{r}\right)
$$

where $q$ is the electron charge, $F$ is the electric field, and $N_{D}$ and $N_{A}$ are the concentrations of ionized donor and acceptor impurities, respectively.

The continuity equations for electrons and holes are:

$$
\frac{\partial n}{\partial t}=+\frac{1}{q} \frac{\partial J_{n}}{\partial x}+U
$$

and

$$
\frac{\partial p}{\partial t}=-\frac{1}{q} \frac{\partial J_{p}}{\partial x}+U
$$

In this work, the organic solar cell is analyzed in steadystate, neglecting transient behaviors. In this case, $\partial n / \partial t=0$ and $\partial p / \partial t=0$ in (5)-(6).

The electron and hole current densities, $J_{n}$ and $J_{p}$, respectively, are controlled by drift and diffusion,

$$
\begin{aligned}
& J_{n}=q n \mu_{n} F+q D_{n} \partial n / \partial x \\
& J_{p}=q p \mu_{p} F-q D_{p} \partial p / \partial x
\end{aligned}
$$

Here, $D_{n}$ and $D_{p}$ are the diffusion coefficients for electrons and holes. The diffusion coefficients are assumed to follow the Einstein relation $D_{n, p} / \mu_{n, p}=V_{T}=k_{B} T / q$ where $k_{B}$ is the Boltzmann constant. In highly disordered systems or semiconductors with large values of the charge carrier density and traps, this relation can be altered $[39,40]$. Several authors propose this relation to be $D_{n, p} / \mu_{n, p}=n V_{T}$, where $n$ is an ideality factor which is induced from trap assisted recombination processes $[5,39]$. Other authors use a carrier-density dependent diffusioncoefficient in the Einstein relation for the transport in Gaussian or Exponential Density of State (DOS) systems [41, 42].

The set of transport equations is completed with the relation between the electrostatic potential $V$ and the electric field:

$$
\partial V / \partial x=-F
$$

From (9), the difference of the electrostatic potential between anode and cathode is

$$
V(0)-V(L)=\int_{0}^{L} F d x=V_{A C}-V_{b i}
$$

where $V_{A C}=V_{\text {anode }}-V_{\text {cathode }}$ is the external applied voltage between anode $(x=0)$ and cathode $(x=L), L$ is the device length, $V_{b i}=\left(\phi_{A}-\phi_{C}\right) / q$ is the built-in voltage and $\phi_{A}$ and $\phi_{C}$ are the work-functions of the metallic contacts of the anode and cathode, respectively.

The total current density in OSCs is given by

$$
J=J_{n}(x, t)+J_{p}(x, t)+\varepsilon_{0} \varepsilon_{r} \partial F(x, t) / \partial t
$$

where the displacement current density $J_{d}=\varepsilon_{0} \varepsilon_{r} \partial E(x, t) / \partial t$ is zero under the quasi-static assumption. Furthermore, since the total current density is uniform in the device, then

$$
\partial J / \partial x=\partial\left(J_{n}+J_{p}\right) / \partial x=0
$$

The system of equations (4)-(12) has been used extensively in the literature to model the transport in OSCs. However, there are particular aspects that differ among different researchers, such as the models used for the charge-carrier generation and recombination, the mobility or the boundary values for the charge carrier concentration, the last one being the main objective of this work.

Regarding the generation rate $G$, which accounts for the optical illumination, this rate is usually assumed constant for simplicity [43, 44]. In our case, the net rate of generated carriers, eq. (3), in combination with TMM, follows Hilczer's and Tachiya's model [36]. In darkness, excitons do not intervene in the generation-recombination processes in the semiconductor, $G=0$, and only bimolecular recombination appears in the semiconductor.

The mobility can depend on variables such as the temperature, the electric field, the charge-carrier concentrations, or the density of states of the organic materials [45-47]. Nevertheless, there exist large ranges of these variables in which the mobility can be assumed constant [48-50]. This assumption simplifies the complex numerical treatments as the ones found in OPV systems. In fact, the mobility in organic diodes, including OPV devices, does not seem to vary much with bias, at low electric fields and low injection of charge $[45,51]$. For these reasons, the mobility is assumed constant in this work. The third aspect that also affects the solution of the transport equations (4)-(12) is the selection of proper boundary conditions, which is the objective of our work and treated in the next section.

\section{Boundary-condition model for OSCs}

\subsection{Boundary-condition model for OSCs in darkness}

The boundary conditions at the OSC contacts represent known values for some physical quantities at the edge of a spatial mesh, in which the integration of the differential equations is performed. At the contacts, the boundary conditions reflect the physics of charge injection and extraction and must match the biasing voltages. The boundary conditions must be chosen to guarantee a self-consistent solution of the numerical model. The basic one-dimension (1-D) semiconductor equations of the OSC active layer (given in the previous section) are solved considering the origin $x=0$ at the anode-semiconductor interface, and the end of the device $x=L$ at the cathode-semiconductor interface. We need as many boundary conditions as the number of differential equations and physical variables is. There are six independent differential equations in (4)-(12) and the same number of physical unknown parameters $\left(V, F, n, p, J_{n}\right.$ and $J_{p}$ ). Two of the boundary values correspond to the potential at the contacts (anode- and cathode-organic interfaces). Zero reference potential is set at the anode, and the potential at the cathode comes from (10), that is 


$$
\begin{array}{r}
V(x=0)=0 \mathrm{~V} \\
V(x=L)=-V_{A C}+V_{b i}
\end{array}
$$

The other four boundary conditions are related to the values of the free charge carrier densities at the anode and cathode of the OSC. As mentioned in the introduction, simple approximations are commonly used in the literature: constant values of the free carrier density, based on the Boltzmann approximation; or constant values of the surface recombination velocity, which account for the ability to transfer current between two regions with different conductions, and determine the interface charge density as a linear function of the current density $J[5,24]$. More accurate models that relate the electric field at the metalorganic interface with the current density can also be found, although their major inconvenience is computational [16, 52], owing to recursion between the transport equations for the OSC active layer and not very simple expressions for the boundary conditions.

A balance between simplicity and accuracy can be found in previous studies we made in darkness in single-carrier and bipolar organic diodes [26]. We observed that the free charge density at the injecting contact of single-carrier and bipolar diodes is related to the current density following the power-law function (1). Fig. 2(a) captures the main conclusions of our previous studies on bipolar organic diodes and their application to OSCs in darkness [26]. It shows the evolution of the free charge density (electrons and holes) at the anode and cathode of an OSC as a function of the current density. This figure points out the following features:

- At forward bias, or positive current densities $\left(J_{f} \equiv J\right)$, the anode injects holes and the cathode injects electrons, the values of the hole density $p_{A}=p(0)$ at the anode and electron density $n_{C}=n(L)$ at the cathode follow (1). The symbols $n, p, A, C$ are related to electrons, holes, anode and cathode, respectively. From experimental observations, the extracted electron and holes densities at the injecting electrodes of bipolar organic diodes are very similar [26]. For this reason, the values of $p_{A}$ and $n_{C}$ can be considered the same: $n_{C}=p_{A}=K_{2}+K_{1} J^{m}$. Another reason to do this is the meaning of the parameters in (1) [27, 28]: $m$ depends on the organic material, being the same for the OSC and $K_{1}$ is related to the energy barrier the charge carriers see when injected towards the organic material. For an OSC with Ohmic contacts, similar energy barriers for electrons and holes are expected at the cathode and anode, respectively. Thus, $K_{1}$ is also expected to be the same for both. The evolution of $p_{A}=n_{C}$ is thus the following. At low current densities, the carrier densities at the injecting contacts are constant $\left(p_{A}=n_{C}=K_{2}\right)$. Once a threshold value of the current density $J_{D}$ is surpassed, the charge density evolves with the current density as $K_{1} J^{m}$.

- At forward bias $\left(J_{f} \equiv J\right)$, the values of the charge densities at the extracting contacts $\left(p_{C}=p(L)\right.$ and $\left.n_{A}=n(0)\right)$ are very small with almost no effect on the simulation results
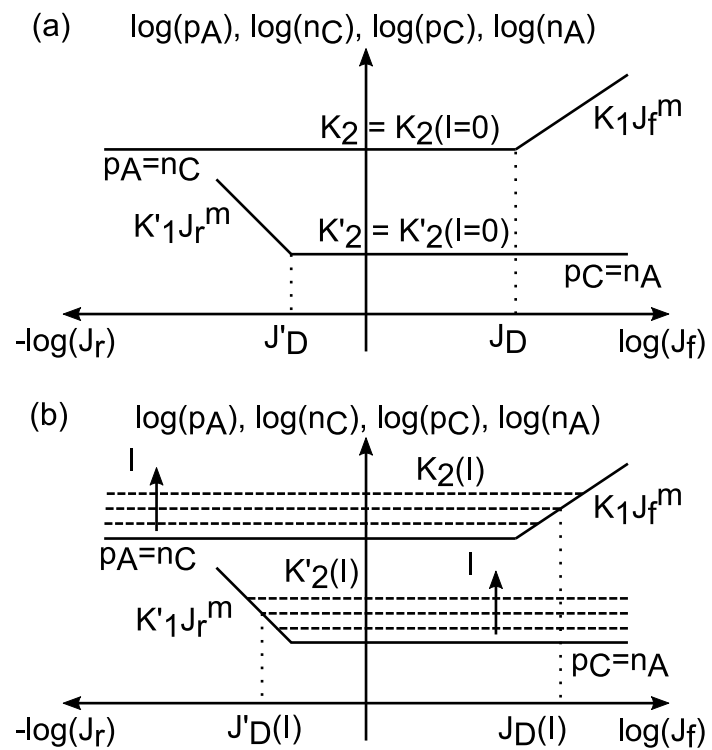

(c)

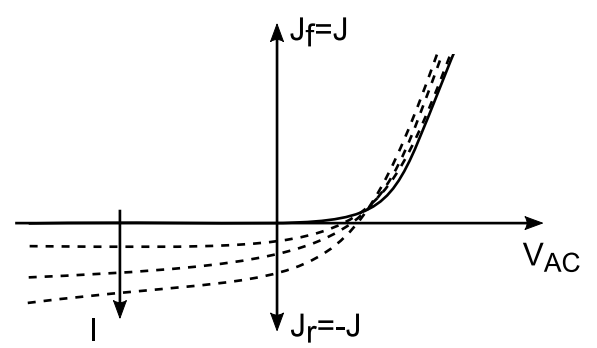

Figure 2: (a) Model for the boundary values of the free charge densities at anode and cathode in an OSC in darkness following the power-law function (14) and Eq.(17). (b) The plateaus in the model elevate under illumination. (c) Typical $J-V_{A C}$ curves of an OSC in dark (solid line) and under different illumination intensities (dashed lines).

$\left(n_{A}=p_{C}=K_{2}^{\prime}\right)$. A relation between $p_{C}$ and $p_{A}$ can be established at low current densities by imposing the condition $J=0$ at zero applied voltage $V_{A C}=0 \mathrm{~V}$. In analytical $J-V_{A C}$ relations for organic diodes (see (A.4) in [26]): $p_{C} \approx p_{A} \times \exp \left(-V_{b i} / V_{T}\right)$, which at low current densities means $K_{2}^{\prime} \approx K_{2} \times \exp \left(-V_{b i} / V_{T}\right)$, where $K_{2}$ and $K_{2}^{\prime}$ are the flat values of $p_{A}$ and $p_{C}$, respectively (see (1) and Fig. 2(a)).

- At reverse bias, or negative current densities $\left(J_{r} \equiv-J\right)$, the roles of the anode and cathode are changed, the anode injects electrons and the cathode injects holes, the values of the hole density $p_{C}=p(L)$ at the cathode and the electron density $n_{A}=n(0)$ at the anode follow (1). For the same reasons as in forward bias, $n_{A}$ and $p_{C}$ are considered the same $\left(n_{A}=p_{C}=K_{2}^{\prime}+K_{1}^{\prime} J_{r}^{m}\right)$. The only difference with forward bias is that holes and electrons see a higher energy barrier at their injecting contacts. The effect of these different energy barriers in forward or in reverse is reflected in different values of the parameter $K_{1}^{\prime} \neq K_{1}$. The value of $m$ in (1) is the same for positive and negative current densities as it only depends on the properties of the organic material [26-28]. 
- At reverse bias $\left(J_{r} \equiv-J\right)$, the values of the carrier densities at the extracting contacts, $p_{A}=p(0)$ and $n_{C}=n(L)$, are constant values and, for consistency, equal to their value in the flat region at $J=0$.

The evolution of the charge densities in Fig. 2(a) shows sharp transitions at the threshold values $J_{D}$ and $J_{D}^{\prime}$. This transition can be softened in order to exactly interpret experimental currentvoltage characteristics of OSCs. In this regard, the parameter $s$ is introduced in the power law model (1),

$$
p_{A}=n_{C}=K_{2}\left\{1+\left[\max \left(0, \frac{J_{f}}{J_{D}}\right)^{m}\right]^{s}\right\}^{\frac{1}{s}}
$$

where $s=1$ for a smooth transition ((14) coincides with (1)) and $s \approx 10$ for sharper transitions. In any case, the powerlaw function is recovered at current densities larger than the threshold current density $J_{D}$

$$
p_{A}=n_{C} \simeq K_{2}\left(\frac{J_{f}}{J_{D}}\right)^{m}=K_{1} J_{f}^{m}, J_{f} \gg J_{D}
$$

with $J_{D}=\left(K_{2} / K_{1}\right)^{(1 / m)}$. The flat region is also reproduced with (14) at current densities lower than the threshold voltage $J_{D}$,

$$
p_{A}=n_{C} \simeq K_{2}, J_{f} \ll J_{D}
$$

An identical relation to (14) can be written for the evolution of $p_{C}=n_{A}$ with $J$, using $p_{C}$ instead of $p_{A}, J_{D}^{\prime}$ instead of $J_{D}$ and $J_{r}=-J$ instead of $J_{f}=J$,

$$
p_{C}=n_{A}=K_{2}^{\prime}\left\{1+\left[\max \left(0, \frac{J_{r}}{J_{D}^{\prime}}\right)^{m}\right]^{s}\right\}^{\frac{1}{s}}
$$

where $J_{D}^{\prime}=\left(K_{2}^{\prime} / K_{1}^{\prime}\right)^{1 / m}$.

\subsection{Boundary-condition model for OSCs under illumination}

The model for the boundary values of the free charge density at the anode and cathode of OSCs ((14) and (17) or Fig. 2(a)) reflects the main conclusions extracted from our previous works in darkness [26-28]. This model is now adapted for OSCs under illumination (Fig. 2(c)), incorporating ideas from other researchers. In studies of light emitting diodes and photovoltaic cells, Malliaras et al. proposed that the free charge densities at the anode and cathode interfaces increase with the illumination [32]. Shuttle et al. measured the stored charge in the active layer of OSCs in darkness and at open circuit voltage $V_{o c}$ for different illumination intensities [29, 30]. They showed that the charge density increases with $V_{o c}$, and consequently with the illumination intensity. They evaluated the current density in darkness as a function of the charge density and the photocurrent as a function of the charge density at $V_{o c}$. They obtained the same evolution in both cases: $J \propto n^{2.6}$. This power-law relation is very similar to our power-law function between the current density and the charge density at the interfaces.

These authors' conclusions under illumination are related to the open circuit voltage region, or current density close to zero. In this regard, we propose that the illumination increases the value of the charge density at the contacts only in the low current region (close to and below the open-circuit voltage). For applied voltages greater than $V_{o c}$, the current density is controlled by drift mechanisms, and the diffusion and illumination play a minor role. Thus, the relation $p_{A}-J$ under illumination is expected to follow our power-law function (15) at high current densities unaltered, as found in dark. With these ideas, the charge density at the contacts under illumination intensity $I$ can be written as:

$$
\begin{aligned}
& p_{A}=n_{C}=K_{2}(I)\left\{1+\left[\max \left(0, \frac{J_{f}}{J_{D}(I)}\right)^{m}\right]^{s}\right\}^{\frac{1}{s}} \\
& p_{C}=n_{A}=K_{2}^{\prime}(I)\left\{1+\left[\max \left(0, \frac{J_{r}}{J_{D}^{\prime}(I)}\right)^{m}\right]^{s}\right\}^{\frac{1}{s}}
\end{aligned}
$$

where $K_{2}(I)=K_{2}+\Delta K_{2}(I), K_{2}^{\prime}(I)=K_{2}^{\prime}+\Delta K_{2}^{\prime}(I), \Delta K_{2}(I)$ and $\Delta K_{2}^{\prime}(I)$ are the densities of photogenerated carriers, $J_{D}(I)=$ $\left(K_{2}(I) / K_{1}\right)^{(1 / m)}$ and $J_{D}^{\prime}(I)=\left(K_{2}^{\prime}(I) / K_{1}^{\prime}\right)^{(1 / m)}$. In darkness $K_{2}(0)=K_{2}$ and $K_{2}^{\prime}(0)=K_{2}^{\prime}$. The evolution of the charge density at the contacts with illumination (18) is depicted in Fig. 2(b). Note that $K_{1}, K_{1}^{\prime}, m$ and $s$ are not altered by the illumination.

The distribution of the photogenerated excitons inside the active layer can be non-uniform according to the TMM results. However, the values of the charge density at the extracting electrodes are expected to be a little smaller than their values at the opposite electrodes $\left(p_{C} \leq p_{A}\right.$ and $\left.n_{A} \leq n_{C}\right)$. In this regard, we can assume $p_{C}=n_{A}=K_{2}^{\prime}(I) \leq K_{2}(I)$, where $K_{2}^{\prime}(I)$ is constant for each value of $I$.

The model still requires evolutions of $K_{2}(I), J_{D}(I)$ and $K_{2}^{\prime}(I)$ with the light intensity, bearing in mind important observations made by Shuttle and collaborators after experimental studies on OSCs [29]: the existence of similar relations between the stored charge density in the active layer of the solar cell with the current density in darkness and between the stored charge density at $V_{o c}$ with the photo-current [29]; and that the stored charge density at $V_{o c}$ is related to the photo-current by a power-law function [29]. That means that the physical process at contacts governing dark-current and photo-current is the same.

In order to model this similar behavior, we consider the set of curves of Figs. 3(a) and (c). Figure 3(c) shows typical $J-V_{A C}$ curves of an OSC under illumination. For an illumination intensity $I$, the maximum value of the reverse current $J_{r}$ is named $J_{s a t}(I)=\max \left[J_{r}\left(V_{A C}, I\right)\right]$. This maximum value corresponds to the total photogenerated current, in which recombination is negligible: $\left.J_{\text {sat }}(I)=q \int_{0}^{L} G(x, I)\right) d x$. We propose to relate $J_{\text {sat }}(I)$ with the free charge density at the interfaces with a similar expression to the " $J>0$ " case (see (14)-(15) and Fig. 3(a)),

$$
\begin{array}{r}
p_{A}=n_{C}=K_{2}(I)=K_{2}+\Delta K_{2}(I) \\
=K_{2}+K_{1}\left[J_{\text {sat }}(I)\right]^{m}
\end{array}
$$

At high illumination intensities, the total charge density can be approximated by the net photogenerated charge density,

$$
p_{A}=n_{C}=K_{2}(I) \simeq \Delta K_{2}(I)=K_{1}\left[J_{\text {sat }}(I)\right]^{m}
$$



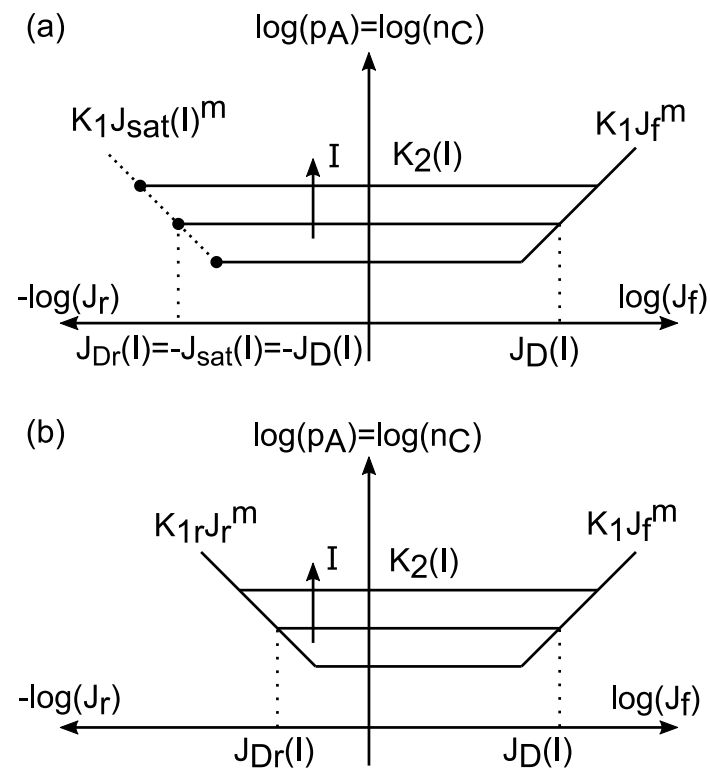

(c)

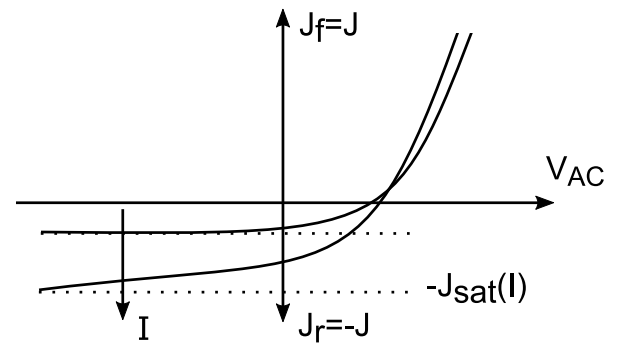

Figure 3: (a) and (b) Graphical representation of (21) for the boundary values of the free charge density at the interfaces of an OSC under illumination (holes at the anode and electrons at the cathode). The injected (right) and extracted (left) charge densities follow a power-law function. Figure (a) represents a similar injection and extraction of charge. Figure (b) shows a dissimilar case, $\left|J_{D r}(I)\right|<\left|J_{D}(I)\right|$, studied in Section 4.3. (c) Typical current-voltage curves of an OSC under illumination.

In a similar way, we might extend the relation (19) to any other variable that produces changes in the value of the free charge density, such as the temperature $T: K_{2}(T)=K_{2}\left(T_{\text {low }}\right)+$ $K_{1}\left(J_{\text {sat }}(T)\right)^{m}$, where $T_{\text {low }}$ is a reference value at low temperatures. The idea that lies beneath our proposals is that the charge density at a contact interface is the one which supports a fixed current through it, no matter the direction and no matter what the origin of the current is. In other words, the value of the current density fixes the value of the charge density at the interface, and vice versa.

The relations (19) and (20) are represented with dotted line in Fig. 3(a). Introducing (19) and (20) in (18), the complete model, including the similar behavior between the injected charge in dark and the extracted charge under illumination, is:

$$
\begin{aligned}
& p_{A}=n_{C}=K_{2}(I)\left\{1+\left[\max \left(\frac{J}{J_{D}(I)}, \frac{J}{J_{D r}(I)}\right)^{m}\right]^{s}\right\}^{\frac{1}{s}} \\
& p_{C}=n_{A}=K_{2}^{\prime}(I) \leq K_{2}(I)
\end{aligned}
$$

with $J_{D r}(I)=-J_{D}(I)=-J_{\text {sat }}(I)<0$ to fulfill the similarity condition, and $K_{2}(I)=K_{1} J_{D}(I)^{m}$.
Equation (21) has three well defined regions. $J_{D}(I)$ is a threshold value of the current density at which the charge densities start to increase over the flat value $K_{2}(I)$ at positive currents (injection regime at forward bias)

$$
p_{A}=n_{C} \simeq K_{2}(I)\left(\frac{J_{f}}{J_{D}(I)}\right)^{m}=K_{1} J_{f}^{m}, J \gg J_{D}(I)
$$

and $J_{D r}(I)$ is a threshold value of the current density at which the charge densities start to increase over the flat value $K_{2}(I)$ at negative currents (extraction regime at reverse bias)

$$
p_{A}=n_{C} \simeq K_{2}(I)\left(\frac{-J_{r}}{J_{D r}(I)}\right)^{m} \equiv K_{1 r} J_{r}^{m}, J \ll J_{D r}(I)
$$

A flat region is found between $J_{D r}(I)$ and $J_{D}(I)$. In this flat region, the value of the charge density at the interfaces depends on the illumination

$$
p_{A}=n_{C} \simeq K_{2}(I), J_{D r}(I) \leq J \leq J_{D}(I) .
$$

$K_{1}=K_{2}(I) /\left[J_{D}(I)\right]^{m}, K_{1 r}=K_{2}(I) /\left[-J_{D r}(I)\right]^{m}$ and $m$ are independent of the illumination, and they describe the power-law trend in the charge injection (22) and extraction (23) at elevating in magnitude bias (see the right and left high current regions in Fig. 3(b)).

\section{Parameter extraction and verification}

\subsection{Parameter extraction and verification - darkness}

In order to validate the boundary-condition model for OSCs in darkness [(14), (17) and Fig. 2(a)], current-voltage curves are calculated with the set of differential equations (4)-(9). In order to reproduce experimental current-voltage curves, the values of the parameters $\mu_{n}, \mu_{p}, V_{b i}, n, K_{1}, K_{1}^{\prime}, m$, and $K_{2}$, are introduced in these equations as follows.

In our calculation, we assume that the carrier mobility in the organic materials is well described by constant values provided in the literature. The nominal value of the built-in potential $V_{b i}$ is given by the difference of the work functions of the electrodes. However, this value can be modified by different effects, such as Fermi-level pinning, spontaneous orientation polarization or band-bending phenomena. There are light-irradiation techniques to estimate $V_{b i}[53,54]$ or the method given by Mantri et al. [55]. We follow this last method, in which an initial value of $V_{b i}$ is extracted from the transition voltage at which the slope of the $J-V_{A C}$ curve changes from exponential (diffusion regime) to power-law (space-charge-limited regime) (point $D$ in Fig. 4). The ideality factor of the Einstein relation $n$ is considered initially as $n=1$. The values of $V_{b i}$ and $n$ can be modified slightly during the extraction of the rest of the parameters related to our model for boundary charge at interfaces.

The extraction procedure depends on the existence or not of parasitic leakage in the OSC, the second case being the most frequent, as this leakage is difficult to avoid. A typical $J-V_{A C}$ curve of an OSC without parasitic leakage in dark is shown with dashed line in Fig. 4(a). The curve can be divided in three regions (I)-(III) in which the above parameters can be extracted in sequential steps. In each step, parameter values are varied 
until a good agreement is obtained between the simulation and the experimental data.

The existence of a parasitic current, such as the one depicted with dotted line in Fig. 4(a), is characterized typically with a shunt resistance $R_{S}$ and symmetric $J-V_{A C}$ behavior. $R_{S}$ has to be taken into account in the extraction procedure, because the leakage current flowing through the shunt resistance $R_{S}$ can mask the low-current regime of the diode. Furthermore, by the presence of $R_{s}$, another region IV also appears in the $J-V_{A C}$ characteristic of OSCs, as shown in Fig. 4(b). Regions I and IV are separated by the corner point $E$. This Fig. 4(b) corresponds to a typical $J-V_{A C}$ curve of an OSC in dark with parasitic leakage.

The sequence of steps needed to extract the parameters of our boundary-charge model for an OSC in dark with parasitic leakage is now detailed.

Step $i\left(K_{2}, K_{2}^{\prime}\right)$ : Regions I and II in the forward bias $\left(J_{f}=J\right)$ show exponential and power-law slopes, respectively, and are separated by the corner point $D\left(V_{b i}, J_{D}\right)$. This corner provides an estimation for the values of $V_{b i}$ and $J_{D}$. The parameter $K_{2}$ is extracted by iteration comparing the experimental data in region I with numerical simulation, imposing boundary conditions $p_{A}=n_{C}=K_{2}$ and $p_{C}=n_{A}=K_{2}^{\prime}$ in the numerical simulation, where $K_{2}^{\prime} \approx K_{2} \times \exp \left(-V_{b i} / V_{T}\right)$ as mentioned in Sec. 2.3.

Step ii $\left(V_{b i}, n, J_{D}\right)$ : The parameters $V_{b i}$ and $n$ (initially $n=1$ ) are varied until the calculated curve agrees with the experimental data in region I. After the fitting of region I, there will be a difference between simulation and experimental data in region II. This difference is greater for larger values of $J_{f}>J_{D}$. A more precise value for $J_{D}$ can be defined at the point in which the experimental and calculated curves differ $5 \%$ in value.

Step iii $\left(m, K_{1}\right)$ : The parameters $K_{1}$ and $m$ are extracted from region II, as they control the high-current regime at forward bias $V_{A C}>V_{b i}$. In this region, use $p_{A}=n_{C}=K_{2}+K_{1} J^{m}$ as boundary charge conditions in the simulations, with $K_{1}=K_{2} / J_{D}^{m}$ and $p_{C}=n_{A}=K_{2}^{\prime}$, and vary $m$ and $K_{1}$ until the calculated curve agrees also with the experimental one in region II.

Step iv $\left(R_{s}\right)$ : In this step, region IV is analyzed. Regions I and IV are separated by the corner point $E$ in Fig. 4(b). The parasitic current component through the shunt resistance $R_{S}$ is calculated as $J_{R s}=V_{A C} /\left(R_{s} A\right)$, where $A$ is the area of the OSC, and compared to the experimental curve in the region IV, providing an initial value for $R_{s}$. Then, the parasitic $J_{R s}$ is added to the current of the intrinsic OSC and compared to the complete $J-V_{A C}$ curve in forward bias (regions I, II and IV). Several iterations may be needed to refine the initial value of $R_{s}$ and obtain a good fitting in region IV.

Step $v\left(K_{1}^{\prime}\right)$ : In this step, the reverse bias region III $\left(J_{r}=-J\right)$ is analyzed. In the comparison between the experimental and calculated curves, use $p_{A}=n_{C}=K_{2}$ and $p_{C}=n_{A}=K_{2}^{\prime}+$ $K_{1}^{\prime} J_{r}^{m}$, where the only unknown parameter to be modified in the fitting process is $K_{1}^{\prime}$. The rest of the parameters were extracted in the previous steps, including $R_{s}$. The total current density in reverse bias (including the parasitic current) is compared to the experimental curve in this region III.

For validation of this procedure, we analyze experimental

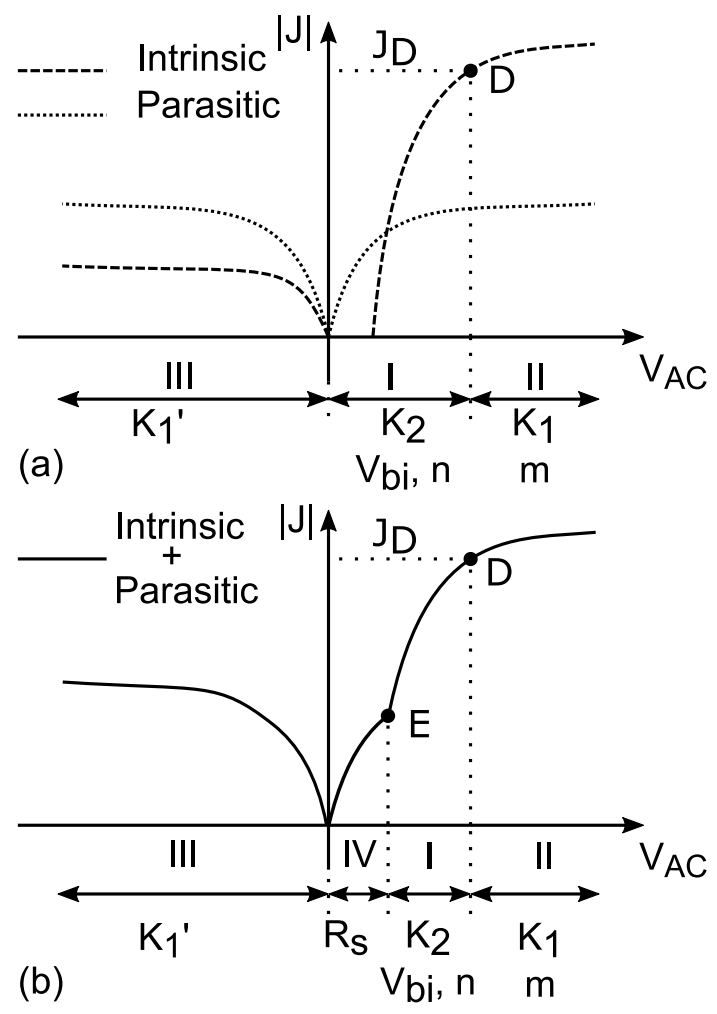

Figure 4: (a) Typical current-voltage curve for an OSC in dark (dashed line) divided into regions I-III in which the parameters of our boundary model (Fig. 2(a)) can be extracted in sequence; and typical parasitic current modeled with a shunt resistance (dotted line). (b) Typical current-voltage curve for an OSC in dark including the parasitic current. In this case, four regions are distinguished, indicating the parameters of the model to be determined in each one.

$J-V_{A C}$ curves of a bulk heterojunction (BHJ) solar cell based on the blend of poly(3-hexylethiophene) (P3HT) and phenyl $[6,6] \mathrm{C}_{61}$ butyric acid methyl ester (PCBM) [56] and depicted with gray circles in Fig. 5. The blend is sandwiched between two ohmic contacts, indium tin oxide (ITO) for holes, and aluminum (Al) for electrons. The complete configuration is ITO/poly(3,4-ethylenedioxythiophene):poly styrene sulfonate (PEDOT:PSS)/P3HT:PCBM/Al.

Figure 5 shows the comparison of these experimental data with our numerical results (solid line). The $J-V_{A C}$ characteristic shows clearly the four regions depicted in Fig. 4(b), including the leakage-current region IV. The values of the free charge-carrier density at the metal-organic interface used in the numerical calculation are shown in Fig. 6. The exponent of the power-law lines in this logarithmic graph is $m=0.35$. The rest of the parameters used in the calculation are $R_{s}=1.5 \mathrm{k} \Omega$, $\mathrm{T}=295 \mathrm{~K}, V_{b i}=0.55 \mathrm{~V}, \mu_{n}=\mu_{p}=10^{-4} \mathrm{~cm}^{2} / V s, n=1.85$, $\varepsilon_{r}=3$, area $A=0.1 \mathrm{~cm}^{2}$ and $L=150 \mathrm{~nm}$. A very good agreement is achieved between the experimental and the calculated curves using our model in dark (14) and (17).

In order to show the effect of the leakage current, a curve without the parasitic $J_{R s}$ of the shunt resistance is shown with dotted line in Fig. 5. The fitting is accurate in regions I and II, but not at low voltages (regions III and IV). It is important to note that the high-current range in region III of reverse biasing 


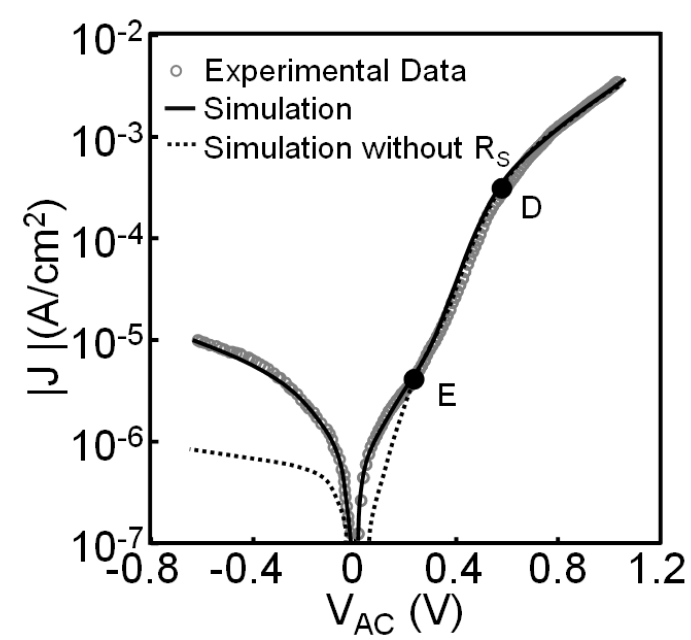

Figure 5: Comparison of an experimental current-voltage curve measured at room temperature in an ITO/PEDOT:PSS/P3HT:PCBM/Al solar cell in dark (symbols)[56] with our numerical results, including the effects of a shunt resistance (solid line) and without these effects (dashed line). The values of the free charge-carrier density at the metal-organic interface used in the numerical calculation are represented in Fig. 6.

cannot be reproduced with the leakage current alone, justifying that Step $v$ was necessary in order to find a proper value for $K_{1}^{\prime}$.

\subsection{Parameter extraction and verification - Under illumination}

Now, the boundary condition model for illumination (21) is tested with experimental $J-V_{A C}$ curves measured in an OSC under different intensities of light. In the numerical simulation, we consider $p_{A}=n_{C}=K_{2}(I)$ from (19), in which the experimental value of $J_{\text {sat }}(I)$ is introduced and $m, K_{1}$ and $K_{2}$ are known from the darkness analysis. We also consider $p_{C}=n_{A}=K_{2}^{\prime}(I) \leq K_{2}(I)$, as was justified in the previous section.

An example of validation of the model under illumination is presented in Fig. 7. In this figure, we compare our numerical results with experimental data measured in the same OSC analyzed in dark (Fig. 5) [56]. A perfect agreement is achieved by introducing the boundary values for the electron and hole densities shown in Fig. 8 in the model of the OSC active layer in Sec. 2. The parameter $K_{2}(I)$, which controls the value of the charge density at the extracting electrodes under illumination, follows the power-law relation (19) with exponent $m=0.35$. This reproduces the same trend observed in darkness at high $J$ (see Fig. 8(a)). The values of the parameter $K_{2}^{\prime}(I)$, which controls the value of the charge density at the opposite electrodes under illumination, are lower than the values of $K_{2}(I)$ (Fig. 8(b)). We have found that their absolute difference is almost constant $K_{2}(I)-K_{2}^{\prime}(I) \simeq 2.4 \times 10^{15} \mathrm{~cm}^{-3}$, meaning that there is a correlation between the boundary values of the charge density under illumination in the two contacts. At high illumination intensities, the relative difference $\left(K_{2}(I)-K_{2}^{\prime}(I)\right) / K_{2}(I)$ decreases. The rest of the parameters coincide with the ones used in darkness.

In order to check that the comparison between the experimental data in Figs. 5 and 7 is not fortuitous, we compared our numerical results with the value of the exponent of the power

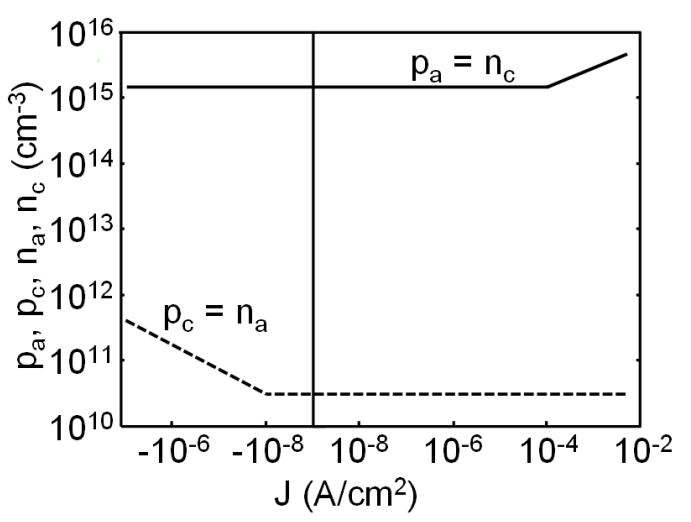

Figure 6: Hole and electron densities at the anode and cathode as a function of the current density, used in the numerical calculation to reproduce the experimental data in Fig. 5. The evolution of $p_{A}=n_{C}$ is described with: $m=0.35$, $J_{D}=10^{-4} \mathrm{~A} / \mathrm{cm}^{2}$ and $K_{2}=1.4 \times 10^{15} \mathrm{~cm}^{-3}$. The evolution of $p_{C}=n_{A}$ is described with: $m=0.35, J_{D}^{\prime}=10^{-8} \mathrm{~A} / \mathrm{cm}^{2}$ and $K_{2}^{\prime}=3 \times 10^{10} \mathrm{~cm}^{-3}$.

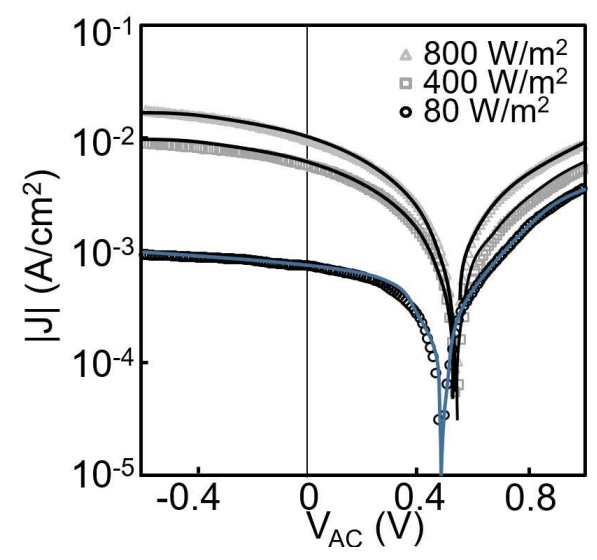

Figure 7: Comparison of experimental current-voltage curves measured at room temperature for an ITO/PEDOT:PSS/P3HT:PCBM/Al solar cell under different radiation intensity (symbols) [56] with our numerical results (solid lines). The values of the free charge-carrier density at the metal-organic interface used in the numerical calculation are presented in Fig. 8.

law extracted from this analysis $(m=0.35)$ with the value proposed in Schuttle's work [29]. They analyzed a similar structure to the one studied in this work. They determined a relation between the stored photogenerated charge density in the bulk and the current density of $n \propto J^{0.38}$. Our power-law model relates the current density with the values of the free charge density at the interfaces. The similarity between the exponent that controls these relations (charge density vs. current density in the bulk or at the interfaces) indicates continuity between bulk and interface charge, and highlights the importance of the use of proper boundary conditions in simulation.

\subsection{Adaptation of the boundary condition model for OSCs with non-ideal contacts}

For an OSC with contacts in which the extraction under illumination is similar to the injection in darkness, our boundary condition model shows symmetry in the charge-current characteristics that is, $\left|J_{D r}(I)\right|=\left|J_{D}(I)\right|$ in Fig. 3(a). This similarity in the model allows for the interpretation of the majority of $J-V_{A C}$ 


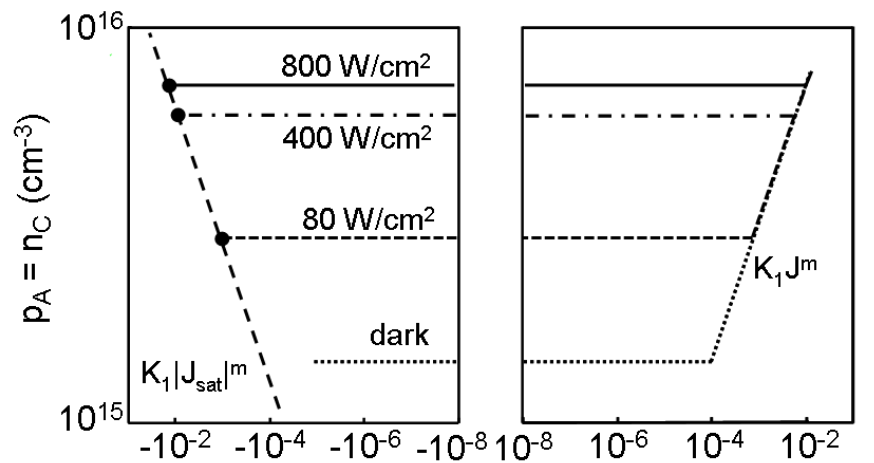

(a)

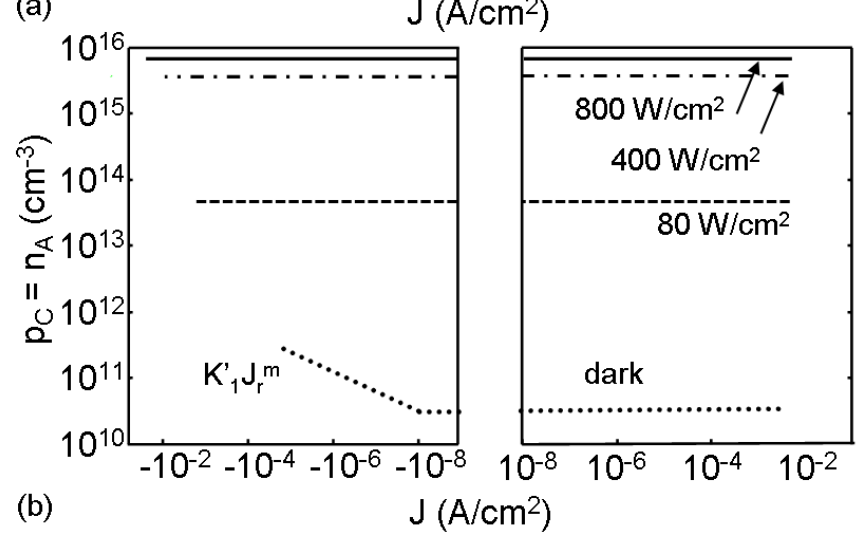

Figure 8: Hole and electron densities at the anode and cathode as a function of the current density. They are used in our numerical procedure to reproduce the experimental data in Fig. 7. (a) The evolution of $p_{A}=n_{C}$ is described with: $m=0.35, K_{1}=3.5 \times 10^{16} \mathrm{~cm}^{2 m-3} \mathrm{~A}^{-m}, J_{D}=10^{-4} \mathrm{~A} / \mathrm{cm}^{2}, K_{2}=$ $1.4 \times 10^{15} \mathrm{~cm}^{-3}, K_{2}\left(80,400,800 \mathrm{~W} / \mathrm{cm}^{2}\right)=2.9,5.9,7.2 \times 10^{15} \mathrm{~cm}^{-3}$. (b) The evolution of $p_{C}=n_{A}$ is described with: $m=0.35, K_{1}^{\prime}=1.9 \times 10^{13} \mathrm{~cm}^{2 m-3} \mathrm{~A}^{-m}$, $J_{D}^{\prime}=10^{-8} \mathrm{~A} / \mathrm{cm}^{2}, K_{2}^{\prime}=3 \times 10^{10} \mathrm{~cm}^{-3}, K_{2}^{\prime}\left(80,400,800 \mathrm{~W} / \mathrm{cm}^{2}\right)=6 \times$ $10^{13}, 3.5 \times 10^{15}, 5.7 \times 10^{15} \mathrm{~cm}^{-3}$.

curves of OSCs with conventional performance, as shown in the preceding section.

In this last section, we analyze what would occur when this similarity is broken and the extraction of charge under illumination follows a trend different from the trend for the injection of charge. This dissimilar situation can be represented using (21) with $\left|J_{D r}(I)\right|<J_{D}(I)$ (see also Fig. 3(b)).

Figure 9 shows a study of the effect of the boundary values for the free charge density at the interfaces $p_{A}=n_{C}$ and $p_{C}=n_{A}$ (Fig. 9(b)) on the $J-V_{A C}$ curves of OSCs under a fixed illumination $I_{0}$ (Fig. 9(a)). Four cases are considered: (i) constant values for $p_{A}=n_{C}$, typical of ideal ohmic contacts using Boltzmann conditions $\left(J_{D}\left(I_{0}\right) \rightarrow \infty, J_{D r}\left(I_{0}\right) \rightarrow \infty\right.$ ); (ii) an OSC with a similar behavior for the injection and extraction mechanisms $\left(J_{D r}\left(I_{0}\right)=-J_{D}\left(I_{0}\right)\right.$ and $\left.K_{1 r}=K_{1}\right)$; (iii) an OSC with a dissimilar behavior for the injection and extraction mechanisms $\left(-J_{D r}\left(I_{0}\right)<J_{D}\left(I_{0}\right)\right.$ and $\left.K_{1}>K_{1 r}\right)$; and (iv) an OSC with a deeper dissimilar behavior $\left(-J_{D r}\left(I_{0}\right) \ll J_{D}\left(I_{0}\right)\right.$ and $\left.K_{1} \gg K_{1 r}\right)$. The value of $p_{C}=n_{A}$ is considered constant and uniform for the four cases, fulfilling the condition $p_{C}=n_{A}=K_{2}^{\prime}\left(I_{0}\right)<K_{2}\left(I_{0}\right)$, which is consistent with our observations of the previous section, and $K_{2}^{\prime}\left(I_{0}\right)$ and $K_{2}\left(I_{0}\right)$ are the values of the flat region of
$p_{C}=n_{A}$ and $p_{A}=n_{C}$, respectively, when the currents through OSCs are low in magnitude.

At $J>0\left(V_{A C}>V_{o c}\right)$, the current density is controlled by the injection of holes and electrons from the anode and cathode, respectively. Their densities at these interfaces are depicted on the right hand side of Fig. 9(b). Case (i) is the only that differs from the rest (ii)-(iv). Case (i) corresponds to constant $p_{A}=n_{C}$ while cases (ii)-(iv) follow the power law at high $J$. Although these differences in boundary conditions for charge seem to be small, the differences in the $J-V_{A C}$ curve are large (Fig. 9(a)), including a qualitative difference in behavior. The results in Fig. 9 magnify the importance of the boundary conditions for the charge in OSCs and confirm the robustness of our powerlaw model to interpret the injection region of the current voltage characteristics of OSCs $\left(V_{A C} \geq V_{o c}\right)$. Actually, the value of $V_{o c}$ can also vary between the different cases of boundary charge in OSCs, as seen in Fig. 9(a).

At $J<0$, the current density in Fig. 9(a) is controlled by the extraction of photogenerated carriers. Cases (i) and (ii) produce the same typical response of an OSC, similar to that studied in Fig. 7. Cases (iii) and (iv) show anomalous S-shape responses, more pronounced in (iv).

These anomalous shapes have been interpreted in the past with low values of the surface recombination velocity $S$ in the boundary relation $J=-S\left(p_{A}-p_{0}\right)[24,48]$, where $p_{A}$ is the hole density at the anode and $p_{0}$ its value at equilibrium (a similar relation can be written for the extraction of electrons at the cathode), or by the introduction of an insulator layer between the metal and organic layers that hinders the flow of the current.

The existence of unintentional doping introduced by oxygen, the presence of traps and impurities or the poor quality of interfacial layers can reduce the velocity at which the carriers are extracted in an illuminated OSC $[24,48]$. In these cases, the accumulation of charge at the extracting electrode elevates, the charge accumulation reduces the value of the photocurrent at $J<0$, and gives rise to anomalous $\mathrm{S}$-shape curves.

Our boundary condition model as defined in (21) can also reproduce these anomalous $\mathrm{S}$-shapes. The accumulation of charge at the extracting electrodes is taken into account with a loss of symmetry between injection and extraction. This dissimilar behavior is controlled in our model (21) with $J_{D r}(I) \neq$ $-J_{D}(I)$ and $K_{1 r} \neq K_{1}$. The reduction of $\left|J_{D r}(I)\right|$ (or the elevation of $\left.K_{1 r}\right)$ make the extracted charge densities at the interfaces $\left(p_{A}, n_{C}\right)$ to increase.

The difference of behavior at $J<0$ among these four curves is explained by the different value of $p_{A}=n_{C}$, which is controlled by the parameter $J_{D r}\left(I_{0}\right)$ (Fig. 9(b)). Case (i) imposes no limitation for the extracted charge with $J_{D r}\left(I_{0}\right) \rightarrow \infty$. Case (ii) does not impose limitation either, it corresponds to contacts that show a similar increment of the injected and extracted charge at high values of $|J|$ because $-J_{D r}\left(I_{0}\right)=J_{D}\left(I_{0}\right)=1 \mathrm{~mA} / \mathrm{cm}^{2}$, and the value of $\left|J_{D r}\left(I_{0}\right)\right|$ is too large to impose a constraint to the extracted charge. Case (iii) with reduced $J_{D r}\left(I_{0}\right)=-10^{-5}$ $\mathrm{mA} / \mathrm{cm}^{2}$ and case (iv) with small $J_{D r}\left(I_{0}\right)=-10^{-7} \mathrm{~mA} / \mathrm{cm}^{2}$ consider an accumulation of charge carriers at very low values of the photocurrent density. After this simulation study, we con- 
clude that the anomalous S-shape curves can be explained by means of a dissimilarity between the extraction and injection of charge at an interface, due to reduced extraction threshold (low $\left|J_{D r}\right|$ ), which causes an increased accumulation of photogenerated charges at the extracting interface at low reverse currents see left-hand side of Fig. 9(b). (a)

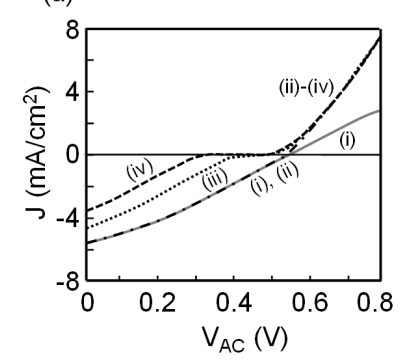

(b)

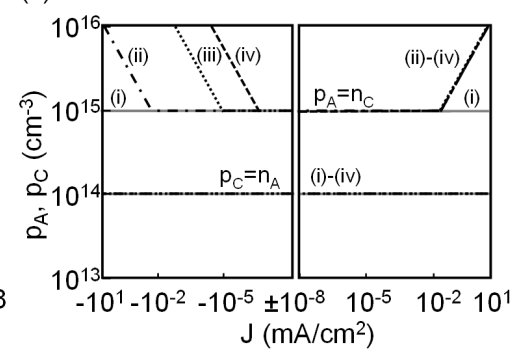

Figure 9: (a) Current-voltage curves of an OSC under a generic illumination $I_{0}$ generated with four different boundaries values for the free charge density $p_{A}=n_{C}$ and $p_{C}=n_{A}$ (named (i)-(iv) in (b)). (b) (i) Ideal extracting contact using Boltzmann conditions $\left(J_{D}\left(I_{0}\right) \rightarrow \infty, J_{D r}\left(I_{0}\right) \rightarrow-\infty\right)$; (ii) contacts with a similar increment of the injected and extracted charge at high values of $|J|$ $\left(\left|J_{D r}\left(I_{0}\right)\right|=J_{D}\left(I_{0}\right)\right)$; (iii)-(iv) contacts imposing limits to the extracted charge. The common parameters used in the simulations are: $\mathrm{T}=295 \mathrm{~K}, V_{b i}=0.55 \mathrm{~V}$, $\mu_{n}=\mu_{p}=10^{-4} \mathrm{~cm}^{2} / \mathrm{Vs}, \varepsilon_{r}=3, L=150 \mathrm{~nm}$ and $s=10$. The specific parameters are: (1) $m=0, K_{2}\left(I_{0}\right)=10^{15} \mathrm{~cm}^{-3}, K_{2}^{\prime}\left(I_{0}\right)=10^{14} \mathrm{~cm}^{-3}$; (2) $m=0.7, K_{2}\left(I_{0}\right)=10^{15} \mathrm{~cm}^{-3},-J_{D r}\left(I_{0}\right)=J_{D}\left(I_{0}\right)=1 \mathrm{~mA} / \mathrm{cm}^{2}$ and $K_{2}^{\prime}\left(I_{0}\right)=$ $10^{14} \mathrm{~cm}^{-3}$; (3) $m=0.7, K_{2}\left(I_{0}\right)=10^{15} \mathrm{~cm}^{-3}, J_{D r}\left(I_{0}\right)=-10^{-5} \mathrm{~mA} / \mathrm{cm}^{2}$, $J_{D}\left(I_{0}\right)=1 \mathrm{~mA} / \mathrm{cm}^{2}$ and $K_{2}^{\prime}\left(I_{0}\right)=10^{14} \mathrm{~cm}^{-3}$; (4) $m=0.7, K_{2}\left(I_{0}\right)=10^{15} \mathrm{~cm}^{-3}$, $J_{D r}\left(I_{0}\right)=-10^{-7} \mathrm{~mA} / \mathrm{cm}^{2}, J_{D}\left(I_{0}\right)=1 \mathrm{~mA} / \mathrm{cm}^{2}$ and $K_{2}^{\prime}\left(I_{0}\right)=10^{14} \mathrm{~cm}^{-3}$.

\section{Conclusions}

In this work, we have addressed the effect of the boundary conditions for the charge density at contact interfaces in the simulation of current-voltage curves of organic solar cells (OSCs) in dark and under illumination. A model for the interface free charge densities at the anode and cathode contacts of OSC has been proposed. The model elaborates a previously developed model for single-carrier and bipolar organic diodes and it is built on experimental observations for OSCs published in the literature. The model relates the free charge density at the interfaces with the current density flowing through the OSC by means of a power-law function. The power-law function can describe both the injection of charge that occurs at voltages greater than the open circuit voltage as well as the extraction of photogenerated charges at voltages lower than the open circuit voltage. The model includes a set of parameters that take into account the operating conditions and features of the contacts in OSCs. Procedures for the extraction of model parameters are also provided. The model has been verified in darkness and under illumination conditions by obtaining a perfect agreement between our numerical results and experimental data published by other authors. The model can also explain anomalous S-shape current-voltage curves through a dissimilarity between injection and extraction. In these anomalous cases, the extracted charge in reverse bias is accumulated at the contact interfaces and hinders the flow of the current.

\section{Acknowledgments}

This work was supported by Ministerio de Educación y Ciencia under research Grant FPU12/02712 and MINECO/FEDER under research Project MAT2016-76892-C3-3-R, and the Canada Research Chair program, NSERC ResEau strategic network and the NCE IC-IMPACTS.

[1] C. Deibel, V. Dyakonov, Polymer-fullerene bulk heterojunction solar cells, Rep. Prog. Phys. 73 (9) (2010) 096401.

[2] R. A. Street, P. P. Khlyabich, B. C. Thompson, Electrical characterization of organic solar cell contact degradation resulting from ambient exposure, Org. Electron. 14 (11) (2013) 2932 - 2939.

[3] M. Jorgensen, K. Norrman, F. C. Krebs, Stability/degradation of polymer solar cells, Sol. Energ. Mat. Sol. C. 92 (7) (2008) 686-714.

[4] R. C. I. MacKenzie, V. S. Balderrama, S. Schmeisser, R. Stoof, S. Greedy, J. Pallarés, L. F. Marsal, A. Chanaewa, E. von Hauff, Loss mechanisms in high efficiency polymer solar cells, Adv. Energy Mater. 6 (2016) 1501742.

[5] A. Petersen, T. Kirchartz, T. A. Wagner, Charge extraction and photocurrent in organic bulk heterojunction solar cells, Phys. Rev. B 85 (2012) 045208 .

[6] J. A. Barker, C. M. Ramsdale, N. C. Greenham, Modeling the currentvoltage characteristics of bilayer polymer photovoltaic devices, Phys. Rev. B 67 (2003) 075205.

[7] L. J. A. Koster, E. C. P. Smits, V. D. Mihailetchi, P. W. M. Blom, Device model for the operation of polymer/fullerene bulk heterojunction solar cells, Phys. Rev. B 72 (2005) 085205.

[8] S. Jung, K.-Y. Kim, Y.-I. Lee, J.-H. Youn, H.-T. Moon, J. Jang, J. Kim, Optical modeling and analysis of organic solar cells with coherent multilayers and incoherent glass substrate using generalized transfer matrix method, Jpn. J. Appl. Phys. 50 (2011) 122301.

[9] A. Mahmoudloo;, S. Ahmadi-Kandjani, Influence of the temperature on the charge transport and recombination profile in organic bulk heterojunction solar cells: a drift-diffusion study, Appl. Phys. A 119: (1523) (2015) 1523-1529.

[10] S. A. Hawks, G. Li, Y. Yang, R. A. Street, Band tail recombination in polymer:fullerene organic solar cells, J. Appl. Phys. 116 (7) (2014) 074503 .

[11] T. Kirchartz, J. Nelson, U. Rau, Reciprocity between charge injection and extraction and its influence on the interpretation of electroluminescence spectra in organic solar cells, Phys. Rev. Appl. 5 (2016) 054003.

[12] Y. Shang, Q. Li, L. Meng, D. Wang, Z. Shuai, Computational characterization of organic photovoltaic devices, Theor. Chem. Acc. 129 (2011) 291-301.

[13] L. Pettersson, L. Roman, O. Inganas, Modeling photocurrent action spectra of photovoltaic devices based on organic thin films, J. Appl. Phys. 86 (1999) 487-496.

[14] P. S. Davids, I. H. Campbell, D. L. Smith, Device model for single carrier organic diodes, J. Appl. Phys. 82 (1997) 6319.

[15] P. Lara Bullejos, J. A. Jiménez Tejada, M. J. Deen, O. Marinov, W. R. Datars, Unified model for the injection and transport of charge in organic diodes, J. Appl. Phys. 103 (2008) 064504.

[16] J. Campbell Scott, G. G. Malliaras, Charge injection and recombination at the metal-organic interface, Chem. Phys. Lett. 299 (2) (1999) 115-119.

[17] J. Campbell Scott, Metal-organic interface and charge injection in organic electronic devices, J. Vac. Sci. Technol. A 21 (2003) 521-531.

[18] V. I. Arkhipov, E. V. Emelianova, Y. H. Tak, H. Bässler, Charge injection into light-emitting diodes: Theory and experiment, J. Appl. Phys. 84 (2) (1998) 848-856.

[19] V. I. Arkhipov, E. V. Emelianova, G. J. Adriaenssens, Effective transport energy versus the energy of most probable jumps in disordered hopping systems, Phys. Rev. B 64 (2001) 125125.

[20] V. I. Arkhipov, P. Heremans, E. V. Emelianova, G. J. Adriaenssens, B. H., Charge carrier mobility in doped disordered organic semiconductors., J. Non-Cryst. Solids 338-340 (2004) 603-606.

[21] V. I. Arkhipov, E. V. Emelianova, A. Kadashuk, H. Bäsler, Hopping model of thermally stimulated photoluminescence organic materials., Chem. Phys. 266 (1) (2001) 97-108.

[22] M. Bouhassoune, S. van Mensfoort, P. Bobbert, R. Coehoorn, Carrierdensity and field-dependent charge-carrier mobility in organic semicon- 
ductors with correlated Gaussian disorder, Org. Electron. 10 (3) (2009) 437-445.

[23] S. L. M. van Mensfoort, R. Coehoorn, Effect of Gaussian disorder on the voltage dependence of the current density in sandwich-type devices based on organic semiconductors, Phys. Rev. B 78 (8) (2008) 085207.

[24] O. J. Sandberg, M. Nyman, R. Österbacka, Effect of contacts in organic bulk heterojunction solar cells, Phys. Rev. Appl. 1 (2014) 024003.

[25] P. López Varo, J. Jiménez Tejada, J. López Villanueva, J. Carceller, M. Deen, Modeling the transition from ohmic to space charge limited current in organic semiconductors, Org. Electron. 13 (9) (2012) 1700 1709

[26] P. López-Varo, J. A. Jiménez-Tejada, O. Marinov, C. H. Chen, M. J. Deen, Charge density at the contacts of symmetric and asymmetric organic diodes, Org. Electron. 35 (2016) $74-86$.

[27] P. López Varo, J. A. Jiménez Tejada, J. A. López Villanueva, M. J. Deen, Space-charge and injection limited current in organic diodes: A unified model, Org. Electron. 15 (10) (2014) 2526 - 2535

[28] P. López Varo, J. A. Jiménez Tejada, J. A. López Villanueva, M. J. Deen, Electrical characterization of controlled and unintentional modified metal-organic contacts, Org. Electron. 15 (10) (2014) 2536-2545.

[29] C. G. Shuttle, A. Maurano, R. Hamilton, B. O’Regan, J. C. de Mello, J. R. Durrant, Charge extraction analysis of charge carrier densities in a polythiophene/fullerene solar cell: Analysis of the origin of the device dark current, Appl. Phys. Lett. 93 (18).

[30] C. G. Shuttle, B. O'Regan, A. M. Ballantyne, J. Nelson, D. D. C. Bradley, J. de Mello, J. R. Durrant, Experimental determination of the rate law for charge carrier decay in a polythiophene:fullerene solar cell, Appl. Phys. Lett. 92 (9) (2008) 093311.

[31] N. Persson, M. Schubert, O. Inganäs, Optical modelling of a layered photovoltaic device with a polyfluorene derivative/fullerene as the active layer, Sol. Energ. Mat. Sol. C. 83 (2004) 169-186.

[32] G. G. Malliaras, J. R. Salem, P. J. Brock, J. C. Scott, Photovoltaic measurement of the built-in potential in organic light emitting diodes and photodiodes, J. Appl. Phys. 84 (3) (1998) 1583-1587.

[33] P. Peumans, A. Yakimov, S. Forrest, Small molecular weight organic thinfilm photodetectors and solar cells, J. Appl. Phys. 93 (2003) 3693.

[34] G. Burkhard, E. Hoke, M. McGehee, Accounting for interference, scattering, and electrode absorption to make accurate internal quantum efficiency measurements in organic and other thin solar cells, Adv. Mater. 22 (2010) 3293-3297.

[35] L. Onsager, Initial recombination of ions, Phys. Rev. 15 (1938) 1

[36] M. Hilczer, M. Tachiya, Unified theory of geminate and bulk electronhole recombination in organic solar cells, J. Phys. Chem. C 114 (2010) 6808-6813.

[37] P. Langevin, The recombination and mobilities of ions in gases, Ann. Chim. Phys. 28 (1903) 433

[38] G. L. A. Rao;, R. H. Friend, Bimolecular recombination in organic photovoltaics, Annu. Rev. Phys. Chem. 65 (557).

[39] C. H. Kim, O. Yaghmazadeh, Y. Bonnassieux, G. Horowitz, Modeling the low-voltage regime of organic diodes: Origin of the ideality factor, J. Appl. Phys. 110 (2011) 093722.

[40] Y. Roichman, N. Tessler, Generalized einstein relation for disordered semiconductors-implications for device performance, Appl. Phys. Lett. 80 (11) (2002) 1948-1950.

[41] S. L. M. van Mensfoort, S. I. E. Vulto, R. A. J. Janssen, R. Coehoorn, Hole transport in polyfluorene-based sandwich-type devices: Quantitative analysis of the role of energetic disorder, Phys. Rev. B 78 (8) (2008) 085208

[42] S. M. Sze, Physics of semiconductor devices, WileyInterscience, 1981.

[43] A. Pivrikas, G. Juška, A. J. Mozer, M. Scharber, K. Arlauskas, N. S. Sariciftci, H. Stubb, R. Österbacka, Bimolecular recombination coefficient as a sensitive testing parameter for low-mobility solar-cell materials, Phys. Rev. Lett. 94 (2005) 176806

[44] A. Wagenpfahl, D. Rauh, M. Binder, C. Deibel, V. Dyakonov, S-shaped current-voltage characteristics of organic solar devices, Phys. Rev. B. 82 (2010) 115306.

[45] R. Coehoorn, W. F. Pasveer, P. A. Bobbert, M. A. J. Michels, Chargecarrier concentration dependence of the hopping mobility in organic materials with Gaussian disorder, Phys. Rev. B 72 (15) (2005) 155206.

[46] B. Ruhstaller, S. Carter, S. Barth, H. Riel, W. Riess, J. C. Scott, Transient and steady state behavior of space charges in multilayer organic light- emitting diodes, J. Appl. Phys. 89 (8) (2001) 4575-4586.

[47] E. Knapp;, B. Ruhstaller, Numerical analysis of steady-state and transient charge transport in organic semiconductor devices, Springer Opt. Quant. Electron. 42 (11) (2011) 667677.

[48] M. Glatthaar, M. Riede, N. Keeganb, K. Sylvester-Hvid, B. Zimmermann, M. Niggemann, A. Hinsch, A. Gombert, Efficiency limiting factors of organic bulk heterojunction solar cells identified by electrical impedance spectroscopy, Sol. Energ. Mat. Sol. C. 91 (2007) 390-393.

[49] M. Neukom, S. Zufle, B. Ruhstaller, Reliable extraction of organic solar cell parameters by combining steady-state and transient techniques, Org. Electron. 13 (12) (2012) 2910-2916.

[50] E. Knapp, B. Ruhstaller, The role of shallow traps in dynamic characterization of organic semiconductor devices, J. Appl. Phys. 112 (2) (2012) 024519-1-6.

[51] R. Häusermann, E. Knapp, M. Moos, N. A. Reinke, T. Flatz, B. Ruhstaller, Coupled opto-electronic simulation of organic bulk-heterojunction solar cells: parameter extraction and sensitivity analysis, J. Appl. Phys. 106 (2009) 104507.

[52] V. I. Arkhipov, U. Wolf, H. Bässler, Current injection from a metal to a disordered hopping system. ii. comparison between analytic theory and simulation, Phys. Rev. B 59 (11) (1999) 7514-7520.

[53] W. Brütting, S. Berleb, A. G. Mükl, Device physics of organic lightemitting diodes based on molecular materials, Org. Electron. 2 (1) (2001) $1-36$.

[54] Y. Noguchi, Y. Miyazaki, Y. Tanaka, N. Sato, Y. Nakayama, T. D. Schmidt, W. Brütting, H. Ishii, Charge accumulation at organic semiconductor interfaces due to a permanent dipole moment and its orientational order in bilayer devices, J. Appl. Phys. 111 (11) (2012) 114508$1-114508-10$

[55] P. Mantri, S. Rizvi, B. Mazhari, Estimation of built-in voltage from steady-state current-voltage characteristics of organic diodes, Org. Electron. 14 (8) (2013) $2034-2038$.

[56] P. Kumar, S. C. Jain, V. Kumar, S. Chand, R. P. Tandon, A model for the J-V characteristics of P3HT:PCBM solar cells, J. Appl. Phys. 105 (10) (2009) 104507. 